\title{
ПАТОЛОГИЯ ШЕЙКИ МАТКИ У ЖЕНЩИН С ЭНДОКРИНОПАТИЯМИ В ПЕРИМЕНОПАУЗЕ
}

\author{
Ужегова Ж.А., Григорян О.Р., Абсатарова Ю.С., Шереметьева Е.В., Михеев Р.К., Андреева Е.Н. \\ ФГБУ «НМИЦ эндокринологии» Минздрава России, Москва
}

ЦЕЛЬ: в связи с развитием новых методов диагностики и лечения предраковых и онкологических заболеваний шейки матки смертность пациенток со злокачественными опухолями этой локализации в последнее время значительно уменьшилась практически во всем мире. Однако данных о распространенности патологии шейки матки среди женщин с различными эндокринными заболеваниями в литературе практически нет, и этот вопрос изучен недостаточно. Проведен сравнительный анализ частоты и характера патологии шейки матки у пациенток перименопаузального периода с различными эндокринопатиями.

МАТЕРИАЛЫ И МЕТОДЫ: в исследование были включены 43 женщины перименопаузального периода (средний возраст 48,8 4,5 года) с различными эндокринными нарушениями: с сахарным диабетом 1 типа - 16 больных; гипофункцией щитовидной железы - 14; функциональной гиперпролактинемией -13.

РЕЗУЛЬТАТЫ: патология шейки матки была выявлена у 73\% женщин с сахарным диабетом 1 типа; у 70,8\% - с гипофункцией щитовидной железы и у 44\% женщин с функциональной гиперпролактинемией. Среди больных с эндокринными нарушениями при цитологическом и гистологическом исследовании в $88 \%$ случаев диагностирован хронический цервицит, на фоне которого у 38,4\% женщин выявлена атипичная кольпоскопическая картина. Анализ гормонального статуса выявил в 86\% случаев гиперэстрогенемию. У 48,7\% женщин с атипичной кольпоскопией диагностирована лейкоплакия шейки матки; у 6,15\% - цервикальная интраэпителиальная неоплазия (ЦИН) различной степени выраженности. У $43 \%$ женщин с функциональной гиперпролактинемией выявлялась комбинация ЦИН, ВПЧ-инфекции и генитального кондиломатоза при сравнении с пациентами с другими эндокринными нарушениями.

Выводы: предварительные результаты исследования показали, что пациенты с эндокринными нарушениями, а также гиперэстрогенемией перименопаузального периода входят в группу риска по возникновению фоновых и предраковых заболеваний шейки матки.

КЛЮЧЕВЫЕ СЛОВА: эндокринопатии; шейка матки; рак шейки матки; эстроген. 